\title{
Andrew Ford, D.D.S.: A Sole-Practitioner Professional Practice Case
}

\author{
Robert M. Cornell, Oklahoma State University, USA
}

Rick Warne, George Mason University, USA

\begin{abstract}
As the U.S. economy continues to shift from manufacturing to service-oriented jobs, students will benefit from exposure to realistic scenarios that service providers may experience. Andrew Ford, D.D.S. is based on a real-life situation facing many single-practitioner professional dental and medical practices. Many professional health care providers have little business experience or training and instead rely on bankers, accountants, and bookkeepers to record accounting entries and provide sound business advice. In this case, students assume the role of a friend/consultant called upon to help determine why the dental practice is not performing at the same level as similar practices. Students encounter a situation in which an overworked medical professional supposes that an employee might be embezzling money or there is some other problem the professional cannot identify, thereby causing low profitability. In this role, the student finds that the professional's accounting records are sparse, making it difficult to precisely evaluate the operation of the practice. The student is asked to analyze the provided information and make suggestions for improving the profitability of the practice. The case also provides students' exposure to basic regression analysis a consultant might use to assess cost behavior. This learning case is appropriate for both undergraduate and graduate managerial accounting classes.
\end{abstract}

Keywords: instructional case; product mix; service industry; cost-volume-profit analysis; managerial decision making; regression analysis

\section{INTRODUCTION}

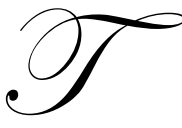

he U.S. economy continues to shift from manufacturing to service-oriented jobs. Accounting research, education, and practice must continue to evolve to provide value-relevant information for economic decisions. Andrew Ford, D.D.S.: A Sole-practitioner Professional Practice Case is based on a real-life situation facing many small businesses in the evolving economy. Many small business owners, including professional health care providers, have little business experience or accounting training. These professionals may initially rely on accounting systems as simple as cash register tapes and check registers to manage their affairs. As the business evolves, the professionals must instead rely on bankers, accountants, bookkeepers, and office managers to provide sound business advice and record accounting entries.

In this case, students assume the role of a friend called upon to help determine why a dental practice is not performing adequately. The student encounters a situation in which a growing business lacks detailed accounting records, making it difficult to accurately evaluate the practice. The student must analyze the available (sparse) information and make suggestions based on assumptions and predictions.

The case also provides students exposure to important cost accounting concepts, requires careful analysis of basic accounting information, and allows students to explore different options - with varying levels of economic risk - when recommending a course of action to the business owner. The case is unique and an important addition to available management accounting case studies because the scenario differs from other cases that rely on precise accounting information. Instead, Andrew Ford, D.D.S. requires students to expand their thinking in search of possible alternatives to determine the cause of low business profitability, and to determine whether the business may be viable going forward. This scenario is accurate to situations faced by many small-business owners, their financial advisors, and accountants. 
We developed our case after much discussion with dentists and with a worldwide dental industry consulting group. Although we simplified the context somewhat for the classroom, our interactions with those in the dental industry indicate that two primary concerns exist: 1) employee embezzlement and fraud, and 2) allocating appropriate time to different procedures (i.e., product mix). Though fraud does not exist in this case, students are required to ascertain whether fraud is a likely cause of the subpar performance of the dentist office. We believe that the principles taught in the case will help students who work as accountants for a service provider or who may consult for such businesses.

\section{CASE MATERIALS}

The following case presents a hypothetical yet realistic scenario that many practitioners face.

\section{Background}

Andrew Ford, D.D.S. is a dentist who owns his own practice located in Edmond, Oklahoma. Andrew's practice is typical since about $75 \%$ of dentists operate their own practices (Bureau of Labor Statistics 2010a). He recently returned from a supplier-sponsored trade show and golf outing in St. Augustine, Florida. While he was there, Andrew spent time learning about new cosmetic dental procedures and networking with other dentists over dinner and on the golf course. This was the first event of this type that Andrew had attended in the last several years.

After lengthy discussions with other dentists who also own small practices, Andrew began to recognize that his practice did not appear nearly as successful as the others. Andrew stayed up late each night worrying about his practice and his future. He realized that although he worked as many hours and had as many patients (likely more) as other dentists, he was not financially as successful as the others.

On the final day of the trip, Andrew attended a seminar designed to teach dentists how to spot embezzlement and fraud at their practices. He learned that according to a recent survey, 59\% of dentists reported experiencing embezzlement during their career (PR.com 2007). Andrew left that session worried that one of his employees might be embezzling from him, which could explain why he was lagging behind his peers financially. He was determined to return to Edmond and ascertain if fraud and theft were causing his practice to suffer or if there was some other problem. As soon as Andrew returned from Florida, he decided he should find someone who might be able to help discover what was causing the poor performance at his practice.

\section{Dental Practice Background}

After earning a degree in Biology, Andrew attended dental school where he graduated at the top of his class. Upon graduation Andrew moved back home to Oklahoma and began working for a local dentist, Dr. James Sutton, in Oklahoma City. Dr. Sutton was a very well-known and respected dentist in the area with a large patient base. Andrew worked long hours as his associate while earning a salary. He thought this experience was necessary to establish himself as a practicing dentist in the area, but the long hours for little reward became frustrating.

Andrew had always planned to open his own practice, and after six years of working for Dr. Sutton, he opened his own dental practice in Edmond, Oklahoma, a small but growing suburb just north of Oklahoma City. The population of Edmond was minimal at the time, but there was promise that this suburb would grow in the future and allow for likely expansion of the practice.

Andrew rented a modest office in a strip mall which also housed a Daylight Donuts, a nail salon, and a dry cleaning business. In the beginning Andrew could only afford one receptionist and one dental hygienist, and he performed a majority of the tasks himself. The receptionist handled all appointments and billing, while the dental hygienist aided in all procedures, from routine cleanings to any special procedures. Andrew worked very hard over the next few years trying to build a successful practice. He ordered all the supplies and saw every patient himself in order to establish a relationship. Partly due to these personal relationships, his patient base grew rapidly and word began to spread throughout the community. 
Within four years of opening, Andrew's practice was booming. He was overrun with patients because of his reputation for quality work and courteous service at a fair price. His practice quickly grew too large for his current staff size, and he recognized the need to hire more employees to handle the increasing patient load. He added another dental hygienist (for a total of two) and assigned each to their own group of patients. In September 2007, he also added a new accounting associate, Jan, to handle the insurance processing and billing. In retrospect, because of his haste in hiring the new personnel, he neglected to follow standard hiring procedures including checking references and conducting background investigations.

\section{Dental Practice Information}

Late in 2008, one of Andrew's suppliers paid for a much needed "working" vacation to the industry trade show to thank him for his loyalty to their company. While playing golf, Andrew began talking to some of the other dentists about how overworked he was, and asked if they were experiencing the same problems. Andrew was shocked to find out that some of the other dentists were not working nearly as many hours as he was while still earning much higher incomes. He could not figure out why this could be since he was working 50 hours per week on average for 50 weeks each year, while some of the other dentists were only working 40 hours per week for the same 50 weeks and making more money than Andrew.

Andrew was tired of consistently working 50 hours a week, and his family life was suffering. Although very willing to work hard, he has always desired a respectable income while working a more normal 40-hour week. Andrew was discouraged and unsure how he can continue to operate his own practice. He even considered returning to a salaried position at a corporate-owned practice that would likely lack opportunity for professional growth.

Andrew decided to call a fraternity brother from college, Peter Campbell, who was working as an accountant at a local firm in Ponca City, to ask for help. During their phone conversation, Andrew told his friend Peter about his recent trip and the final session he attended on embezzlement. He then said, "Peter, I've thought this over and over. I know that my practice should be doing just as well, or better, than these other dentists. Like clockwork, I work ten hours a day, five days a week. I only take a total of two weeks of vacation and personal time away from the practice each year, but I'm still not making any money. I can hardly pay the bills and I'm suffering from working so much but I can't figure out what I'm doing wrong. My family hardly sees me. I'm worried that one of my employees is stealing from me and I think I know who it is. My new accounting assistant, Jan, must be the culprit. Can you help me prove it?

Although he had not heard from Andrew in a few years and had little expertise in investigating fraud and theft, Peter was concerned and agreed to help. Peter visited the practice the next day over his lunch break and began to ask questions and gather information. In addition, Andrew provided Peter with his financial information for the past three years. The financial information revealed that Andrew grouped together all operating costs as "overhead" (this is common for this type of small practice). Although he is certain that the costs in total are accurate, he has difficulty determining where he should place each "overhead" cost on formal financial statements. Table 1 provides three years of financial information for the practice. Table 2 provides the number of procedures performed, sales revenue, and "overhead" expenses per month for the year 2008.

\section{TABLE 1}

Andrew Ford, D.D.S. Income Statements for Years 2006 - 2008

\begin{tabular}{llll} 
& $\underline{2006}$ & $\underline{2007}$ & $\underline{2008}$ \\
Sales Revenue & $\$ 612,095$ & $\$ 622,522$ & $\$ 634,146$ \\
Overhead & $(\$ 562,359)$ & $(\$ 560,269)$ & $(\$ 567,454)$ \\
Operating Income & $\$ 49,736$ & $\$ 62,253$ & $\$ 66,692$ \\
\hline \hline
\end{tabular}


TABLE 2

Andrew Ford, D.D.S. Practice Details Calendar Year 2008

\begin{tabular}{|c|c|c|c|c|}
\hline Month & $\underline{\text { Number of Procedures }}$ & $\underline{\text { Sales Revenue }}$ & Overhead & Operating Income \\
\hline January & 622 & $\$ 52,598$ & $\$ 47,252$ & $\$ 5,346$ \\
\hline February & 635 & $\$ 53,695$ & $\$ 47,498$ & $\$ 6,197$ \\
\hline March & 660 & $\$ 55,799$ & $\$ 47,998$ & $\$ 7,801$ \\
\hline April & 622 & $\$ 52,588$ & $\$ 47,252$ & $\$ 5,336$ \\
\hline May & 628 & $\$ 53,107$ & $\$ 47,377$ & $\$ 5,730$ \\
\hline June & 622 & $\$ 52,604$ & $\$ 47,248$ & $\$ 5,356$ \\
\hline July & 615 & $\$ 51,992$ & $\$ 47,127$ & $\$ 4,865$ \\
\hline August & 622 & $\$ 52,580$ & $\$ 47,252$ & $\$ 5,328$ \\
\hline September & 609 & $\$ 51,495$ & $\$ 46,698$ & $\$ 4,797$ \\
\hline October & 615 & $\$ 52,005$ & $\$ 47,127$ & $\$ 4,878$ \\
\hline November & 628 & $\$ 53,097$ & $\$ 47,373$ & $\$ 5,724$ \\
\hline December & $\underline{622}$ & $\$ 52,587$ & $\$ 47,252$ & $\$ 5,335$ \\
\hline Total & 7,500 & $\$ 634,146$ & $\$ 567,454$ & $\$ 66,692$ \\
\hline
\end{tabular}

\section{TABLE 3}

\section{Industry Comparative Data for Offices of Dentists - NAICS Code 621210*} Annual Revenues of \$350,000 - \$750,000 for years 2006 - 2008

\begin{tabular}{|c|c|c|c|}
\hline & $\underline{\text { Bottom Tercile }}$ & $\underline{\text { Middle Tercile }}$ & Top Tercile \\
\hline Sales & 1.00 & 1.00 & 1.00 \\
\hline Cost of Goods Sold & 0.06 & 0.05 & 0.06 \\
\hline Gross Margin & 0.94 & 0.95 & 0.94 \\
\hline Selling and Administrative Expenses & 0.80 & 0.72 & 0.58 \\
\hline Operating Income $* *$ & 0.14 & 0.23 & 0.36 \\
\hline
\end{tabular}

* Also valid for SIC Code 8021

** Does not include compensation provided to practice owners

Note: NAICS 621210 (Offices of Dentists) comprises establishments of health practitioners having the degree of D.M.D. (Doctor of dental medicine), D.D.S. (Doctor of dental surgery), or D.D.Sc. (Doctor of dental science) primarily engaged in the independent practice of general or specialized dentistry or dental surgery. These practitioners operate private or group practices in their own offices (e.g., centers, clinics) or in the facilities of others, such as hospitals or HMO medical centers. They can provide either comprehensive preventive, cosmetic, or emergency care, or specialize in a single field of dentistry (United States Census Bureau 2010).

\section{Business Analysis}

Peter quickly realized that he would have difficulty determining whether Jan, or anyone else, was embezzling money from the practice without a full investigation. When done properly, such an investigation would cost thousands of dollars and take months. Peter decided, instead, to focus his efforts on his area of financial expertise and then, if necessary, focus on the embezzlement issue later. 
Peter decided to first examine some of the pertinent business issues associated with small professional health care practices. He decided to investigate Andrew's practice and some competing dental practices in the area to determine their revenue structure. He also considered comparing the practice's profitability with industry standards. Industry information is provided in Table 3.

After reviewing the practice, Peter concluded that Andrew was focusing almost solely on low-end services, such as simple teeth cleanings and minor fillings. This allowed Andrew to see more patients each day, but this part of the practice seemed to consume all of Andrew's time. Peter investigated the competing dental practices in the area and visited with representatives from an industry trade group. Most dentists generate their revenue from three distinct areas. The first service offered is simple teeth cleaning and minor fillings, essentially what Andrew was doing exclusively during the day. The second service is tooth restoration / major repair work, and the third is all other cosmetic procedures, including whitening and veneers. The industry trade group suggested that $10 \%$ of all cleanings should result in a restoration / major repair visit. Andrew currently did not spend any time performing either of these other services.

Peter decided to further investigate how the mix of these additional services affected bottom-line profitability of a practice. He found information concerning standards for each new type of procedure (restorative/major repair and cosmetic) from the industry trade group. Revenue and expense information for each procedure is found in Table 4.

\section{TABLE 4}

\section{Proposed Revenue / Expense for Enhanced Procedures}

Restoration / Major Repair ${ }^{1}$

Restoration / Major repair revenue per procedure

Restoration / Major repair materials cost per procedure

Cosmetic Procedure $^{2}$

Cosmetic revenue per procedure

Cosmetic materials cost per procedure

$\$ 300$

Additional Information:

${ }^{1}$ Each restoration / major repair requires 30 minutes of dentist time

${ }^{2}$ Each cosmetic procedure requires 60 minutes of dentist time

\section{An Improved Business Plan?}

Peter explained his findings to Andrew, and the two of them started working together to find ways to improve the bottom line of the practice. After doing some market research, they considered raising prices for simple cleanings and fillings in an attempt to free up time for more profitable procedures. They determined that the average current price was likely too low, and that for every $10 \%$ price increase in preventative care cost, the practice would lose $10 \%$ of the current procedures (i.e., the number of procedures performed annually decreases $10 \%$ for every 10 $\%$ price increase). It also appeared that a 50\% price increase from the current average rate would be the maximum before all patients would seek other providers.

The industry trade group explained that $10 \%$ of the total preventative procedures performed should result in restoration and/or major repair procedures, which are currently not happening at all. These are the first procedures Andrew will perform if there is any free time. Each major restoration/repair takes 30 minutes to complete. Therefore, for every hour freed up by increasing prices, Andrew can perform two restoration procedures. 
Furthermore, if there is any additional free time after the cleanings and the restorations, Andrew can perform the more lucrative cosmetic procedures. These procedures require an hour per procedure, but they are the most profitable. However, there is significant risk associated with these procedures as expensive advertising must be purchased to motivate demand. The only way to improve demand is to purchase additional advertising in the local newspapers, magazines, and billboards. Ultimately, it is risky to spend extra money on advertising without increasing the number of cosmetic procedures. This is just one of the decisions Andrew must make when planning for the future. The number of expected cosmetic procedures based on $\$ 600$ of yearly advertising expenditures is provided in Table 5.

\section{TABLE 5}

\section{Expected Values of Advertising Cost for Cosmetic Procedures}

Advertising is purchased in $\$ 50$ monthly increments for 12 months $=\$ 600$ per year yielding the following expected values of numbers of procedures:

$10 \%$ Probability of 7.5 procedures $=0.75$ Expected Number of Procedures

$20 \%$ Probability of 5.0 procedures $=1.00$ Expected Number of Procedures

$30 \%$ Probability of 2.5 procedures $=0.75$ Expected Number of Procedures

$40 \%$ Probability of 0.0 procedures $=\underline{0.00}$ Expected Number of Procedures

Total Expected Procedures $=2.50$ for $\$ 600$ of annual advertising expense

\section{DISCUSSION QUESTIONS}

The information provided in this case is typical of the quality of information obtained with analyzing many small professional practices. Much of the information requires estimating and inferences. Student responses will vary and rounding is necessary to evaluate the data and respond to discussion questions.

1. Did Andrew properly assess the direct material, direct labor, and overhead costs associated with operating his practice?

2a. Based on your evaluation of the practice in

2b. Based on when Jan was hired, does it appear that reported practice performance declined after her hire, indicating embezzlement?

3a. List the types of fixed, variable, and mixed costs a dental practice would likely incur when providing cleaning procedures.

3b. Plot the sales revenue on an scattergraph with the number of monthly procedures on the $\mathrm{X}$-axis and the monthly sales revenue on the Y-axis. Does it appear that there is a linear relationship between the number of procedures and sales revenue? Prepare a scattergraph for overhead expenses by plotting the number of monthly procedures on the $\mathrm{X}$-axis and the monthly overhead expenses on the $\mathrm{Y}$-axis. Does it appear that there is a linear relationship between the number of procedures and overhead expenses?

3c. Using simple regression, determine the variable cost per procedure and the fixed cost per month for the practice.

3d. What is the coefficient of determination for the regression analysis and what does it mean?

3e. What are the $t$-statistics and $p$-values associated with the fixed and variable costs? How do you interpret the $t$-statistics and $p$-values?

3f. Revenue per procedure varies slightly in medical practices depending on payment methods including cash, credit cards, and insurance reimbursement. Assuming average revenue per existing procedure, what is the contribution margin per procedure? How many procedures must Andrew perform to break-even? How long does it take to perform one existing procedure, and how many hours would it take to perform the necessary procedures to break-even? Does it seem reasonable for one dentist to work that many hours and complete that many procedures?

4a. If the annual target profit (before tax) for the practice is $\$ 150,000$, how many procedures would Andrew have to perform? 
4b. How many hours would it take to perform that many procedures? Does it seem reasonable for one dentist to work that many hours and complete that many procedures?

4c. Based on your answers to $3 a-3 e$ and $4 a-4 b$, compute the $90 \%$ and $95 \%$ confidence intervals (see Table 6 for required $t$-statistics) for the total cost associated with the number of procedures to earn an annual target profit (before tax) of $\$ 150,000$. What does the confidence interval mean?

5. What would be the new price and contribution margin for each $10 \%$ price increase (each increase is an additional $10 \%$ of the initial revenue per procedure, not a compound increase) up to a maximum $50 \%$ price increase. Market research has shown that a price increase in excess of 50\% would cause all customers to seek new dentists.

6a. Assume that Andrew wants to work 40 hours per week for 50 weeks each year (or 2,000 hours per year total). How much does the price per existing procedure need to increase so that Andrew has enough free time to perform other procedures due to the reduction in patients? What are your suggestions of ways to fill this time? Andrew will perform all existing cleaning and major / restorative procedures first and then perform any cosmetic procedures.

6b. Assuming Andrew has moderate risk-tolerance, provide a forward-looking Contribution Income Statement to demonstrate planned practice performance with this service mix. Note: Assume that the variable cost per procedure determined in $3 \mathrm{a}$ is based on 20 minutes of dentist time and would recur with these other procedures as well (i.e. there are no specific costs associated with the cleaning procedures that would not recur with other procedures). Again, Andrew will perform all existing cleaning and major / restorative procedures first and then perform any cosmetic procedures.

6c. Now assume Andrew has high risk-tolerance, provide a forward-looking Contribution Income Statement to demonstrate planned practice performance with this service mix. Note: assume that the variable cost per procedure determined in $3 \mathrm{a}$ is based on 20 minutes of dentist time and would recur with these other procedures as well (i.e. there are no specific costs associated with the cleaning procedures that would not recur with other procedures). Again, Andrew will perform all existing cleaning and major / restorative procedures first and then perform any cosmetic procedures.

7. Assume (independent of all prior assumptions in the case) that Andrew wants to spend $33.33 \%$ of his time on cleanings, $33.33 \%$ of his time on restoration / major repair, and $33.33 \%$ of his time on cosmetic procedures. He is planning a $40 \%$ price increase to allow time to complete these new procedures and is willing to spend $\$ 60,000$ this year on advertising to promote the cosmetic procedures. How many of each type of procedure would Andrew have to complete annually in order to break even? How many hours would it take for Andrew to complete the break-even number of procedures?

8. Provide a memo explaining your findings and present your recommendations. Address the initial concern from Andrew Ford that a staff member was embezzling from him. Carefully explain the problem Andrew is facing due to the mix of services he offers and how changing the product mix will affect practice profitability. Use calculations from your analyses to support your assertions. Provide a business option based on Andrew's moderate and high risk tolerances from $6 \mathrm{~b}$ and $6 \mathrm{c}$. In particular, address the risks of incurring additional fixed costs such as advertising expense. Carefully explain any potential downside risk associated with incurring additional fixed advertising costs.

\section{CASE LEARNING OBJECTIVES AND IMPLEMENTATION GUIDANCE}

In the ten-year span from 2009-2018, manufacturing jobs in the United States are expected to decline by 9\% despite the projected growth in population. In contrast, service industries are expected to add 14.5 million jobs in this same time period (Bureau of Labor Statistics 2010b). As most students will likely find employment in the service industry, they will benefit by participating in learning activities that provide examples of situations that service providers may encounter.

Andrew Ford, D.D.S. introduces and combines several management accounting concepts leading to improved business design and practices. This case is based on a real-life situation facing many service providers who have little formal business training and instead rely on others to provide sound business advice. The student assumes the role of a friend who is asked to help determine the reasons a dental practice is not profitable. In this role, the student finds that the professional's accounting records are sparse, making it difficult to precisely evaluate the operation of the practice. The student is asked to analyze the provided information and make suggestions for 
improving the profitability of the practice.

This case links topics that are typically covered in separate chapters in many management accounting courses and thereby provides an opportunity to associate these topics in a real-life situation to which many students can easily relate. The linked topics include cost behavior including fixed, variable, and mixed costs; product pricing; regression analysis and interpretation; contribution margin; expected values; break-even and target profit analyses; product mix decisions; and pro forma contribution format income statements. Perhaps one of the most important components of the case is that certain questions require students to evaluate risky alternatives and suggest a course of action for the naïve business owner. This scenario provides an opportunity for students to arrive at different conclusions with different answers based on their own perspectives instead of attempting to arrive at one "correct" answer.

To successfully address the case requirements, students should possess a basic understanding of accrual accounting concepts including revenues, expenses, assets, liabilities, and the format of traditional financial statements including the balance sheet and the income statement. Also, students should possess the ability to communicate well in writing as the case requires the composition of a business memo. Instructors may perceive the required memo as the most important component of the case as it requires the student to communicate financial information, including risks associated with suggested changes in the business, to a naïve business owner with little accounting knowledge. The memo requirement is designed to reinforce the accounting concepts by requiring students to explain their recommendations in a detailed, organized manner.

The instructor can choose to select all or parts of the discussion questions to highlight specific topics of interest. The following specific learning objectives should be achieved by completing all of the case requirements: 1) Understand how to incorporate simplistic revenue and cost data in a small professional service firm; 2) Gain an understanding of cost behavior; 3) Develop product mix recommendations using expected values of expenditures; 4) Complete a recommendation memo including recommendations and analyses directed to a naïve business owner; 5) Integrate basic statistical analysis into a management accounting context.

\section{Suggested Audience and Implementation Guidance}

The primary audience for this case consists of students in any accounting course in which performance measurement, information analysis, critical thinking, and written communication are examined. Students in introductory management accounting courses at most universities will likely find the case requirements challenging. The case is most appropriate for junior-level or senior-level accounting courses as well as MBA courses. Examples of classes that may find this case useful include higher-level undergraduate management accounting course, an entrepreneurship course dealing with small business or professional practice issues, or a management accounting course at the graduate level such as an MBA management accounting course. A suggestion for appropriate questions for these students follows.

To prove relevant in many types of accounting courses, the case provides questions that vary in difficulty. Question difficulty levels are identified with the solution in the Teaching Notes. Questions labeled easy or medium would be appropriate for use in an introductory undergraduate managerial accounting course. Questions labeled easy, medium, or difficult would be appropriate for a higher-level undergraduate managerial accounting course or a graduate-level managerial accounting course (e.g., MBA-level managerial accounting).

\section{Student Evaluation of the Case}

We tested the case in one section of intermediate managerial accounting students (i.e., students had completed an introductory managerial accounting course in a prior academic year) and with two sections of MBA managerial accounting. A total of 98 students participated in the case evaluation. After submitting the case for course credit, the instructor distributed a questionnaire to assess the extent to which the case achieved the desired learning objectives and to obtain general student reaction to the case. 
In general, we found that the MBA students found this case more challenging because they needed significantly more instruction in interpretation of statistical results than did the undergraduate accounting students. The MBA students indicated that their formal statistics training had occurred primarily as undergraduates and that integrating basic statistical concepts into the case scenario was helpful.

\section{ACKNOWLEDGEMENT}

We thank John H. Jameson, D.D.S. of Jameson Comprehensive Coaching for providing important details related to a small dental practice such as the one described in this case. We also appreciate the Risk Management Association for allowing us to adapt the format of their annual statement studies for use in the context of this case. We are grateful for the helpful comments we received from Robert Allen. Finally, we thank the graduate students who provided important feedback on earlier versions of this case.

\section{AUTHOR INFORMATION}

Robert M. Cornell, $\mathrm{PhD}, \mathrm{CMA}$ is an assistant professor of accounting at Oklahoma State University. Prior to earning his $\mathrm{PhD}$ at the University of Utah, Professor Cornell worked as a controller for a commercial / industrial construction company and as a bank commercial loan officer. Professor Cornell conducts research and writes case studies on a variety of management and cost accounting topics. In his free time, he enjoys spending time with his family and serving with the local affiliate of Habitat for Humanity.

Rick C. Warne, PhD, CPA is an assistant professor of accounting at George Mason University. He graduated with his $\mathrm{PhD}$ degree from the University of Utah and earned his MAcc and BS degrees in accounting from Brigham Young University. Professor Warne worked at the Office of the Utah State Auditor prior to entering academia. He has published both academic articles and articles of interest to practitioners in a variety of outlets. Professor Warne is also a Certified Public Accountant in the Commonwealth of Virginia.

\section{REFERENCES}

1. Bureau of Labor and Statistics, 2010a. Occupational Outlook Handbook, 2010-2011. http://www.bls.gov/oco/ocos072. htm. Accessed April 5, 2010.

2. 2010b. Occupational Outlook Handbook, 2010-2011. http://www.bls.gov/oco/oco2003.htm. Accessed April 7, 2010.

3. PR.com, 2007. Most Dentists Report Being Embezzled: The Wealthy Dentist Survey Results. http://pdf.pr.com/press-release/pr-37247.pdf. Accessed April 5, 2010.

4. United States Census Bureau, 2010. 2002 NAICS Definitions: 621210 Offices of Dentists. http://www.census.gov/epcd/naics02/def/ND621210.htm. Accessed April 5, 2010. 


\section{TEACHING NOTES}

The case provides discussion questions that vary in their difficulty. Question difficulty levels are identified with the solution. Questions labeled easy or medium would be appropriate for use in an introductory undergraduate managerial accounting course. Questions labeled easy, medium, or difficult would be appropriate for a higher-level undergraduate managerial accounting course or a graduate-level managerial accounting course (e.g., MBA-level managerial accounting).

\section{Solutions to Discussion Questions}

1. Did Andrew properly assess the direct material, direct labor, and overhead costs associated with operating his practice?

Andrew does not correctly account for materials, labor, or overhead costs when developing financial statements or analyzing business profitability. This is a common problem in many small professional practices and other small businesses. In many cases, all costs are conceptualized as being "overhead" or simply the cost of operating the business. With small professional practices the practitioners profit is often regarded as the remaining income after expenses.

Question level $=$ easy

2a. Based on your evaluation of the practice in comparison to industry averages, does it appear that the practice is operated profitably?

Referring to the industry tercile data given in Table 2, and applying it to the practice data given, this practice is operating at less than the bottom tercile. This shows that the practice is simply not profitable when compared to peer firms. Clearly something needs to be changed to provide a market wage to the dentist and a return on his investment in the practice.

\section{Question level $=$ easy}

2b. Based on when Jan was hired, does it appear that reported practice performance declined after her hire, indicating embezzlement?

Jan was hired in September of 2007. Upon examination of the provided data for the last three years ('06'08) the practice is virtually unchanged in terms of revenues and costs. The practice has been unprofitable for quite some time now, and the hiring of Jan towards the end of 2007 does not change that. There is little evidence of embezzlement by Jan. There is, however, significant evidence of an inefficiently operated dental practice.

\section{Question level = easy}

3a. List the types of fixed, variable, and mixed costs a dental practice would likely incur when providing cleaning procedures.

Possible fixed costs of operating a dental practice include a lease or depreciation on the practice building, lease or depreciation on equipment, insurance, and property taxes. Variable costs would include supplies / materials used in procedures and off-site laboratory fees. Mixed costs would include staff labor, utilities, and maintenance on the building and equipment.

Question level = easy

3b. Plot the sales revenue on an scattergraph with the number of monthly procedures on the $\mathrm{X}$-axis and the monthly sales revenue on the Y-axis. Does it appear that there is a linear relationship between the number of procedures and sales revenue? Prepare a scattergraph for overhead expenses by plotting the number of 
monthly procedures on the $\mathrm{X}$-axis and the monthly overhead expenses on the $\mathrm{Y}$-axis. Does it appear that there is a linear relationship between the number of procedures and overhead expenses?

The preparation of scattergraphs allows the student to visualize the relationship between the number of procedures and both sales revenue and overhead expenses. The scattergraphs shown in Exhibit 1 indicate a strong relationship between procedures and both sales revenue and overhead expenses that can be further investigated through regression analysis.

Exhibit 1 - Scattergraphs for Sales Revenue and Overhead Expenses

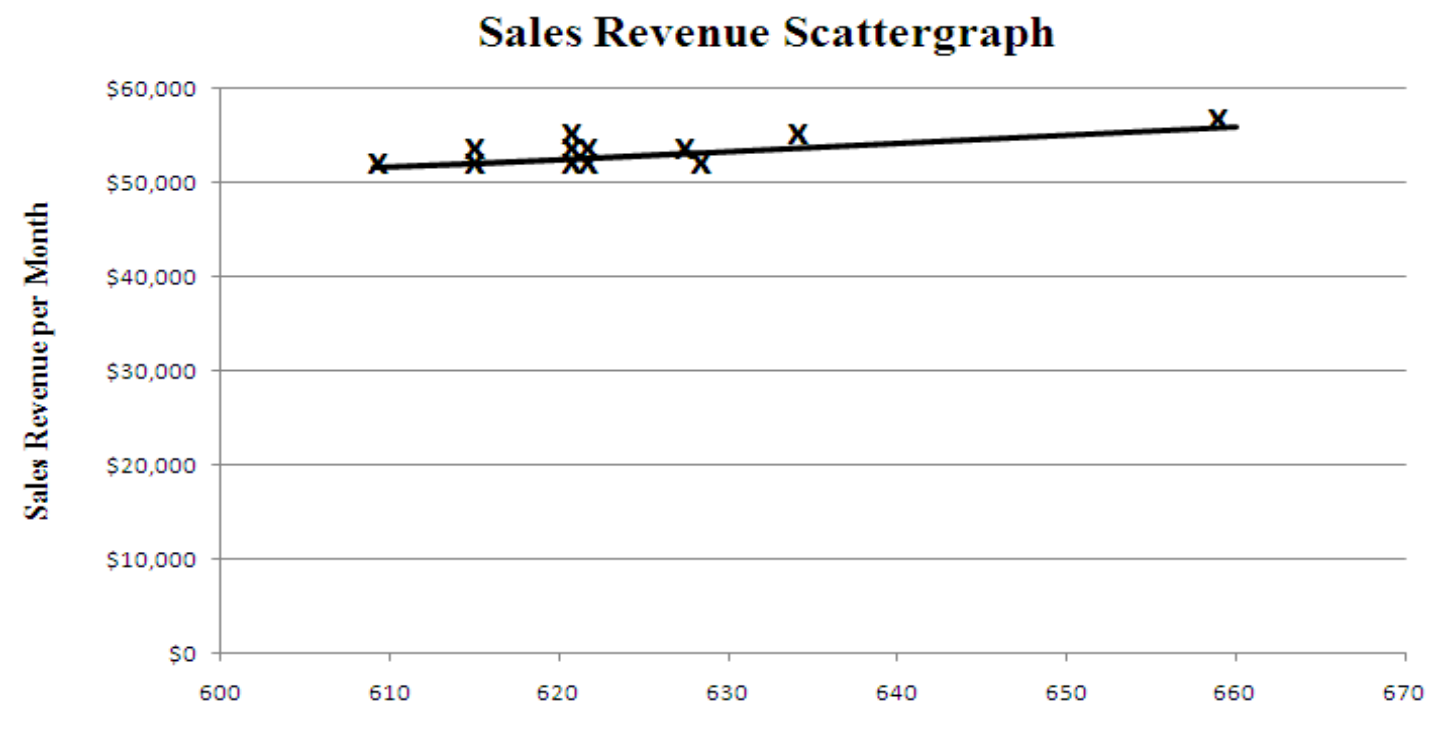

Procedures per Month

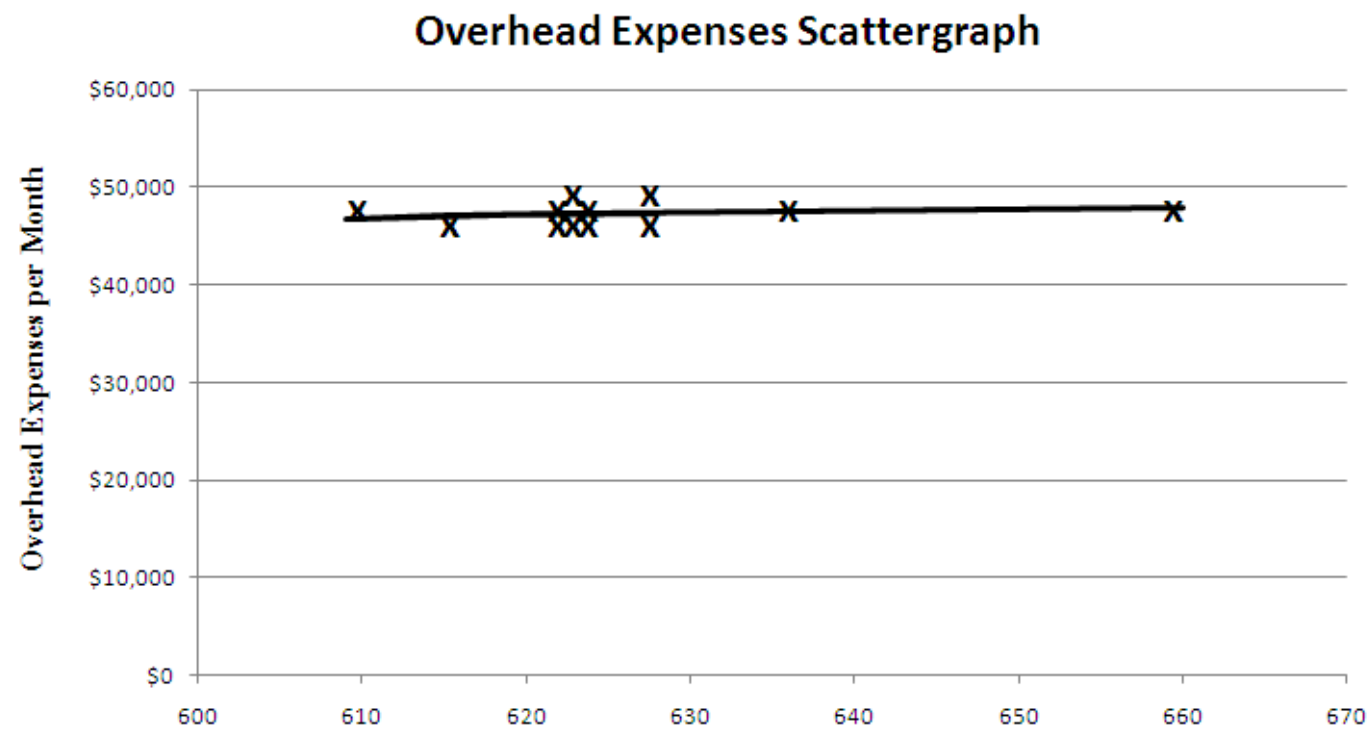

Procedures per Month 
Question level = easy

3c. Using simple regression, determine the variable cost per procedure and the fixed cost per month for the practice.

Students should refer to the information provided on Table 2 to respond to this question. The simple regression analysis yields the following results (Exhibit 1 - Regression Analysis Results provides complete regression results):

\author{
Primary Figures - Regression Results \\ Intercept $=\$ 33,498.52$ (rounded) \\ Slope Variable $=\$ 22.06$ (rounded) \\ $R$ Square $=0.925$
}

Exhibit 2 - Regression Analysis Results

\begin{tabular}{lc}
\hline \multicolumn{2}{c}{ Regression Statistics } \\
\hline Multiple R & 0.962 \\
R Square & 0.925 \\
Adjusted R square & 0.918 \\
Standard Error & 85.126 \\
Observations & 12.000 \\
\hline
\end{tabular}

\begin{tabular}{|c|c|c|c|c|c|}
\hline & $d f$ & $S S$ & $M S$ & $F$ & Significance $p$ \\
\hline Regression & 1 & 897607.297 & 897607.297 & 123.869 & 0.000 \\
\hline Residual & 10 & 72464.369 & 7246.437 & & \\
\hline Total & 11 & 970071.667 & & & \\
\hline & Coefficients & Standard Error & $t$ Stat & $P$-value & \\
\hline Intercept & 33498.517 & 1239.217 & 27.032 & 0.000 & \\
\hline $\mathrm{X}$ Variable 1 & 22.063 & 1.982 & 11.130 & 0.000 & \\
\hline
\end{tabular}

Question level $=$ medium

3d. What is the coefficient of determination for the regression analysis and what does it mean?

The coefficient of determination measures the goodness of fit of the data points to the resulting regression line. The coefficient of determination for these data is 0.925 , the $R$ square $\left(R^{2}\right)$ value. The coefficient of determination ranges from 0 to 1.00 and statistically measures the percentage of the total variance of the dependent variable that is explained by the independent variable. In this case, the model is the relationship between the number of procedures and total cost to operate the dental practice.

Question level $=$ medium

3e. What are the $t$-statistics and $p$-values associated with the fixed and variable costs? How do you interpret the $t$-statistic and $p$-value?

Hypothesis tests of parameters test whether the parameters (fixed cost and variable cost) are significantly different from zero. The $t$ statistics (equal to coefficients divided by standard errors for each parameter) and associated p-values indicate the level of significance for each parameter. The closer the p-value is to zero, the more likely it is that the true parameter is significantly different from zero. Conventionally, p-values of 0.05, 0.01, and 0.001 are standards used to determine significance.

In this case, both p-values are less than 0.001 indicating that both the true fixed and true variable costs are significantly different than zero. 
t-statistic for Fixed Cost $=33,498.517 / 1239.217=27.032, p$-value $=0.000$

$t$-statistic for Variable Cost $=22.063 / 1.982=11.130, p$-value $=0.000$

Question level $=$ medium

3f. Revenue per procedure varies slightly in medical practices depending on payment methods including cash, credit cards, and insurance reimbursement. Assuming average revenue per existing procedure, what is the contribution margin per procedure? How many procedures must Andrew perform to break-even? How long does it take to perform one existing procedure, and how many hours would it take to perform the necessary procedures to break-even? Does it seem reasonable for one dentist to work that many hours and complete that many procedures?

After completing the regression analysis, students should obtain variable cost per procedure equaling $\$ 22.06$. In addition, the average revenue per procedure of $\$ 84.55$ (\$634,146 Annual Sales Revenue / 7,500 procedures) yields a contribution margin of $\$ 62.49$ per procedure. With fixed costs totaling $\$ 33,498.52$ per month Andrew must perform 536 procedures per month or 6,432 procedures per year to break-even. The text of the case indicates that the dentist works 50 hours per week for 50 weeks per year for a total of 2,500 hours per year. Table 2 shows that he completed 7,500 procedures during that time for time per procedure of 20 minutes (7,500 procedures / 2,500 hours $=3$ procedures per hour or 20 minutes per procedure). Assuming 20 minutes per procedure this number of procedures would require 2,144 hours per year or 41.2 hours per week assuming 52 full weeks of work per year excluding holidays, vacation, or other time off. Clearly, this is not reasonable to expect from one professional.

Question level $=$ medium

4a. If the annual target profit (before tax) for the practice is $\$ 150,000$, how many procedures would Andrew have to perform?

Andrew must cover $\$ 33,498.52$ in fixed costs per month $\times 12$ months $=\$ 401,982.24$ plus the desired profit of $\$ 150,000=\$ 551,928.24$. To achieve that, Andrew would need to perform $(\$ 551,928.24 / \$ 62.49)=8,833$ (rounded) procedures per year.

Question level $=$ medium

4b. How many hours would it take to perform that many procedures? Does it seem reasonable for one dentist to work that many hours and complete that many procedures?

Assuming each procedure requires 20 minutes of dentist time, completing that number of procedures would require 2,944 hours of work per year for the dentist. It does not seem reasonable that the dentist would be able to work that much for a long period of time. This level of output would require 56.6 hours of work per week for 52 weeks a year excluding holidays, vacation, or other time off.

\section{Question level $=$ medium}

4c. Based on your answers to $3 a-3 d$ and $4 a$, compute the $90 \%$ and $95 \%$ confidence intervals (see Table 6 for $t$-statistics) for the total cost associated with the number of procedures to earn an annual target profit (before tax) of $\$ 150,000$. What does the confidence interval mean?

Confidence intervals provide an upper and lower-bounded range of values for the actual cost with a prespecified degree of confidence. Confidence intervals allow managers to predict a range of values that will result based on a predicted level of the activity driver. The confidence interval is calculated as:

[Fixed Cost $+($ Variable Cost per procedure * Number of procedures)] $\pm t$-statistic (Standard Error for the model $)=($ lower bound, upper bound $)$ 
The total cost associated with 8,833 procedures is:

$$
\begin{aligned}
& \$ 401,982.24+\$ 22.063(8,833 \text { procedures })=\$ 596,864.72 \\
& \text { The confidence intervals for that cost are }(10 \text { degrees of freedom }): \\
& 85 \%(\text { two-sided })=\$ 596,864.72 \pm 1.090(85.126)=(\$ 596,771.93, \$ 596,957.51) \\
& 90 \%(\text { two-sided })=\$ 596,864.72 \pm 1.372(85.126)=(\$ 596,747.93, \$ 596,981.51) \\
& 95 \%(t w o-s i d e d)=\$ 596,864.72 \pm 1.812(85.126)=(\$ 596,710.47, \$ 597,018.97) \\
& 99 \%(t w o-\text { sided })=\$ 596,864.72 \pm 2.764(85.126)=(\$ 596,629.43, \$ 597,100.01) \\
& 99.9 \%(\text { two-sided })=\$ 596,864.72 \pm 4.144(85.126)=(\$ 596,511.96, \$ 597,217.48)
\end{aligned}
$$

\section{$\underline{\text { Question level }=\text { medium }}$}

5. What would the new price and contribution margin be for each $10 \%$ price increase up to a maximum $50 \%$ price increase (each increase is an additional $10 \%$ of the initial revenue per procedure, not a compound increase). Market research has shown that a price increase in excess of $50 \%$ would cause all customers to seek new dentists.

$\begin{array}{llllll}\text { Price Increase } & 10 \% & 20 \% & 30 \% & 40 \% & 50 \% \\ \text { New Price } & \$ 93.01 & \$ 101.46 & \$ 109.92 & \$ 118.37 & \$ 126.83 \\ \text { (Variable Cost) } & \$ 22.06 & \underline{\$ 22.06} & \$ 22.06 & \$ 22.06 & \$ 22.06 \\ \text { Contribution Margin } & \$ 70.95 & \$ 79.40 & \$ 87.86 & \$ 96.31 & \$ 104.77\end{array}$

Question level $=$ medium

6a. Assuming Andrew wants to work 40 hours per week for 50 weeks each year, at what percent price increase increment does Andrew begin to have free time? What are your suggestions of ways to fill this time? Andrew will perform all existing cleaning and major / restorative procedures first and then perform any cosmetic procedures.

It's not until Andrew raises his prices by $30 \%$ on cleanings does he begin to have free time. By raising his price to $\$ 109.92$ he is projecting to lose 2,250 patients, thus clearing some time to do other procedures such as the restoration and cosmetic procedures.

Question level $=$ difficult

6b. Assuming Andrew has moderate risk-tolerance, provide a forward-looking Contribution Income Statement to demonstrate planned practice performance with this service mix. Note: assume that the variable cost per procedure determined in $3 \mathrm{a}$ is based on 20 minutes of dentist time and would recur with these other procedures as well (i.e. there are no specific costs associated with the cleaning procedures that would not recur with other procedures). Again, Andrew will perform all existing cleaning and major / restorative procedures first and then perform any cosmetic procedures.

See Price Increase Solution Table Below (Answers will vary but will likely center around $20 \%-30 \%$ price increase). Contribution margins for the new procedures are:

Restoration / Major Repair Revenue

Restoration / Major Repair Variable Cost Materials

Existing Variable Cost / Procedure * 1.5

Contribution Margin per Procedure

Cosmetic Procedure Revenue

Cosmetic Procedure Variable Cost Materials

Existing Variable Cost / Procedure *3

Contribution Margin per Procedure
$\$ 650$

$\$ 150$

$\$ 33.09$

$\$ 466.91$

$\$ 1,200$

$\$ 300$

$\$ 66.19$

$\$ 833.81$ 


\section{Question level $=$ medium}

6c. Now assume Andrew has high risk-tolerance, provide a forward-looking Contribution Income Statement to demonstrate planned practice performance with this service mix. Note: assume that the variable cost per procedure determined in $3 \mathrm{a}$ is based on 20 minutes of dentist time and would recur with these other procedures as well (i.e. there are no specific costs associated with the cleaning procedures that would not recur with other procedures). Again, Andrew will perform all existing cleaning and major / restorative procedures first and then perform any cosmetic procedures.

See Exhibit 2 Price Increase Solutions below and Contribution Margins from $6 b$ (Answers will vary but will likely center around 40\% - 50\% price increase). Note that enough increase in fixed advertising expense will need to be incurred to support the projected increase in cosmetic procedures. Table 5 shows that each $\$ 600$ in annual advertising cost yields an expected 2.5 cosmetic procedures.

Advertising is purchased in $\$ 50$ monthly increments for 12 months $=\$ 600$ per year yielding the following expected values of numbers of procedures:

$10 \%$ Probability of 7.5 procedures $=0.75$ Expected Number of Procedures

$20 \%$ Probability of 5.0 procedures $=1.00$ Expected Number of Procedures

$30 \%$ Probability of 2.5 procedures $=0.75$ Expected Number of Procedures

$40 \%$ Probability of 0.0 procedures $=0.00$ Expected Number of Procedures

Total Expected Procedures $=2.50$ for $\$ 600$ of annual advertising expense

\section{See Exhibit 3}

Question level $=$ difficult

7. Assume (independent of all prior assumptions in the case) that Andrew wants to spend $33.33 \%$ of his time on cleanings, $33.33 \%$ of his time on restoration / major repair, and $33.33 \%$ of his time on cosmetic procedures. He is planning a $40 \%$ price increase to allow time to complete these new procedures and is willing to spend up to $\$ 60,000$ this year on advertising to promote the cosmetic procedures. How many of each type of procedure would Andrew have to complete annually in order to break even? How many hours would it take for Andrew to complete the break-even number of procedures?

At a price increase level of $40 \%$ the contribution margin for each cleaning / minor repair is $\$ 96.31$. The contribution margin for each restoration / major repair is $\$ 466.91$ and for each cosmetic procedure is $\$ 833.81$ (both from discussion question 6b). The new fixed cost per year is $\$ 401,982.20+\$ 60,000$ (additional advertising) $=$ $\$ 461,982.20$. The simplest solution is to create a three-hour bundle of services including three cleaning / minor repairs, two major / restoration, and one cosmetic procedure. The total contribution margin for this bundle is $\$ 2,056.56$ for three hours of dentist time. To cover fixed costs the dentist must perform 224.64 (225 rounded) bundles or 673.92 hours ( 674 hours) of work. This work would include 673.92 (674 rounded) cleanings, 449.28 (450 rounded) major repairs / restorations, and 224.64 (225 rounded) cosmetic procedures.

Question level $=$ difficult

8. Provide a memo explaining your findings and present your recommendations. Address the initial concern from Andrew Ford that a staff member was embezzling from him. Carefully explain the problem Andrew is facing due to the mix of services he offers and how changing the product mix will affect practice profitability. Use calculations from your analyses to support your assertions. Also, address the risks of incurring additional fixed costs such as advertising expense will affect anticipated profitability going forward. Carefully explain potential downside risk associated with incurring additional fixed advertising costs. 
Responses to the required memo will vary depending on the insights of each student. Clearly, there are higher risks associated with the greater rewards of advertising and performing the cosmetic procedures. These lucrative procedures are advertising-driven and could dramatically increase the profitability of the practice. However, advertising is not a sure bet and the expected values of number of procedures realized for advertising dollars spent could be affected by general economic factors, changing trends, or the selection of appropriate advertising outlets. In general, it is clear that something must be done to improve the business practices associated with Andrew Ford. The recommendations made in this memo should include increasing pricing to allow for free time to pursue more lucrative business, risks associated the potential increase in fixed costs necessary to pursue the strategy, and a discussion of the risk / reward payoff associated with suggestions. The memo allows the instructor to grade not only students' quantitative skills but also their ability to communicate (complex) financial analysis in a written format that is understandable by a naïve professional. Due to the comprehensive nature of this question, instructors may desire to place more grading emphasis (points assigned to this question) than on other portions of the case.

Question level $=$ medium 


\begin{tabular}{ccc}
$10 \%$ & $20 \%$ & $30 \%$ \\
$\$ 93.01$ & $\$ 101.46$ & $\$ 109.92$ \\
$\$ 22.06$ & $\$ 22.06$ & $\$ 22.06$ \\
$\$ 70.95$ & $\$ 79.40$ & $\$ 87.86$ \\
6750 & 6000 & 5250 \\
20.00 & 20.00 & 20.00 \\
135000 & 120000 & 105000 \\
2250 & 2000 & 1750 \\
$(250.00)$ & 0.00 & 250.00 \\
& & \\
675.00 & 600.00 & 525.00 \\
0.50 & 0.50 & 0.50 \\
337.50 & 300.00 & 262.50 \\
$(587.50)$ & $(300.00)$ & $(12.50)$ \\
0 & 0 & 500 \\
0 & 0 & 0 \\
& & \\
$\$ 627,804.59$ & $\$ 608,780.21$ & $\$ 577,072.91$ \\
$\$ 148,924.62$ & $\$ 132,377.44$ & $\$ 115,830.26$ \\
$\$ 478,879.97$ & $\$ 476,402.77$ & $\$ 461,242.65$ \\
$\$ 0.00$ & $\$ 0.00$ & $\$ 325,000.00$ \\
$\$ 0.00$ & $\$ 0.00$ & $\$ 91,547.18$ \\
$\$ 0.00$ & $\$ 0.00$ & $\$ 233,452.82$ \\
$\$ 0.00$ & $\$ 0.00$ & $\$ 0.00$ \\
$\$ 0.00$ & $\$ 0.00$ & $\$ 0.00$ \\
$\underline{\$ 0.00}$ & $\underline{\$ 0.00}$ & $\underline{\$ 0.00}$ \\
$\$ 478,879.97$ & $\$ 476,402.77$ & $\$ 694,695.47$ \\
& & \\
$\$ 401,982.20$ & $\$ 401,982.20$ & $\$ 401,982.20$ \\
$\$ 0.00$ & $\$ 0.00$ & $\$ 0.00$ \\
$\underline{\$ 401,982.20}$ & $\underline{\$ 401,982.20}$ & $\$ 401,982.20$ \\
$\underline{\$ 76,897.77}$ & $\underline{\$ 74,420.57}$ & $\underline{\$ 292,713.27}$ \\
\hline & & \\
\hline & & \\
& &
\end{tabular}

\begin{tabular}{cc} 
& Exhibit $3-$ Price Incre \\
$40 \%$ & $50 \%$ \\
$\$ 118.37$ & $\$ 126.83$ \\
$\$ 22.06$ & $\$ 22.06$ \\
\hline$\$ 96.31$ & $\$ 104.77$ \\
4500 & 3750 \\
20.00 & 20.00 \\
90000 & 75000 \\
1500 & 1250 \\
500.00 & 750.00 \\
& \\
450.00 & 375.00 \\
0.50 & 0.50 \\
225.00 & 187.50 \\
275.00 & 562.50 \\
450 & 375 \\
275.00 & $562.50 *$ \\
& \\
$\$ 532,682.68$ & $\$ 475,609.54$ \\
$\$ 99,283.08$ & $\$ 82,735.90$ \\
$\$ 433,399.60$ & $\$ 392,873.64$ \\
$\$ 292,500.00$ & $\$ 243,750.00$ \\
$\$ 82,392.46$ & $\$ 68,660.39$ \\
$\$ 210,107.54$ & $\$ 175,089.61$ \\
$\$ 330,000.00$ & $\$ 675,000.00$ \\
$\$ 100,701.90$ & $\$ 205,981.16$ \\
$\$ 229,298.10$ & $\$ 469,018.84$ \\
$\$ 872,805.24$ & $\$ 1,036,982.10$ \\
& \\
$\$ 401,982.20$ & $\$ 401,982.20$ \\
$\$ 66,000.00$ & $\$ 135,000.00$ \\
$\$ 467,982.20$ & $\$ 536,982.20$ \\
$\$ 404,823.04$ & $\$ 499,999.90$ \\
\hline & \\
\hline &
\end{tabular}

\section{ease Solutions}

Price increase percentage

New price

Variable cost per procedure

New contribution margin

Procedures

Time in minutes per procedure

Total minutes for this many procedures

Total hours for this many procedures

Hours free for other procedures

Number of expected restoration / major repair

Hours necessary for each restoration / major repair

Hours necessary for expected restoration / major repair

Hours free minus hours necessary for expected restoration / major repair

Number of restoration / major repair predicted to perform

Number of cosmetic predicted to perform

Revenue from existing procedures

Variable cost from existing procedures

Contribution margin from existing procedures

Revenue from Major / Restorative

Variable cost from Major / Restorative

Contribution margin from Major / Restorative

Revenue from Cosmetic

Variable cost from Cosmetic

Contribution margin from Cosmetic

Total Contribution Margin from all procedures

Total Fixed Cost from before

Additional fixed cost for advertising

Total Fixed Cost

Operating Income

* It is impossible to perform one-half of a procedure. Student responses for the $50 \%$ price increase will likely be 562 or 563 procedures with slight differences (as compared to this solution) in revenue, cost, and income due to this difference. 


\section{NOTES}

